\title{
ON THE INVARIANTS OF DIFFERENTIAL FORMS OF DEGREE HIGHER THAN TW0*
}

BY

\section{CHARLES NELSON HASKINS}

\section{§1. Introduction.}

The following determination of the number of invariants of homogeneous differential forms of higher degree is, in notation and method, an extension of the work in my paper on the invariants of quadratic differential forms. $\dagger$

The result may be stated in the following

Theorem.-The number of invariants of order $\mu$ for the general homogeneous differential form of degree $m$ in $n$ variables is

$$
\frac{(n+\mu-1) !}{(n-1) ! \mu !}\left\{\frac{(n+m-1) !}{(n-1) ! m !}-\frac{n(n+\mu)}{\mu+1}\right\}, \quad m>2 .
$$

It may be noted that, save for the exceptional cases

$$
n \equiv 2, \mu=0,1 \text {, or } 2 \text {; } \quad \text { and } \quad n=2, \mu=3 \text {; }
$$

this expression gives also the number of invariants of quadratic differential form $(m=2) \cdot \ddagger$

§2. Differential equations of the problem.

The form of degree $m$ in $n$ variables, $x_{1} \cdots x_{n}$, may be written

where

$$
\phi \equiv \sum_{i_{1}=1}^{n} \sum_{i_{2}=1}^{n} \cdots \sum_{i_{m}=1}^{n} a_{i_{1} i_{2} \ldots i_{m}} d x_{i_{1}} d x_{i_{2}} \cdots d \dot{i_{i_{m}}}
$$

$$
a_{i_{1} i_{2} \ldots i_{m}}=a_{k_{1} k_{2} \ldots k_{m}},
$$

if $i_{1}, \cdots i_{m} ; k_{1}, \ldots k_{m}$ are any two permutations of the same set of $m$ indices* It will be assumed throughout that the discriminant of the form $\phi, i$. e., the resultant of the $n$ equations

- Presented to the Society October 25, 1902. Received for publication October 29, 1902.

† Haskins, Transactions of the American Mathematical Society, vol. 3 (1902), pp. 71-91.

$\ddagger$ loc. cit., p. 89. 
does not vanish.

$$
\frac{\partial \phi}{\partial\left(d x_{r}\right)}=0, \quad(r=1,2, \ldots n)
$$

The form is to be subjected to the infinite group of all point transformations generated by the infinitesimal transformation

$$
X f \equiv \sum_{r=1}^{n} \xi_{r}\left(x_{1} \cdots x_{n}\right) \frac{\partial f}{\partial x_{r}} .
$$

To obtain the equations satisfied by the invariants, we must "extend" the group $X f$ to the coefficients $a$ and their derivatives. As in the similar work with quadratic forms, we use the notation

$$
\begin{aligned}
\alpha_{i_{1} i_{2} \ldots i_{m}} & =X\left(a_{i_{1} i_{2} \ldots i_{m}}\right) \\
p_{i_{1} i_{2} \ldots i_{m}} & =\frac{\partial f}{\partial\left(a_{i_{1} i_{2} \ldots i_{m}}\right)}, \\
\xi_{r \mid l} & =\xi_{r}=\frac{\partial \xi_{r}}{\partial x_{l}} .
\end{aligned}
$$

Then we have

$$
X \phi=\sum_{i_{1}=1}^{n} \sum_{i_{2}=1}^{n} \cdots \sum_{i_{m}=1}^{n} \alpha_{i_{1} i_{2} \ldots i_{m}} d x_{i_{1}} d x_{i_{2}} \cdots d x_{i_{m}}
$$

$$
\begin{aligned}
& +\sum_{i_{1}=1}^{n} \sum_{i_{2}=1}^{n} \cdots \sum_{i_{m}=1}^{n} a_{i_{1} i_{2} \ldots i_{m}} \sum_{l=1}^{m} d x_{i_{1}} \cdots d x_{i_{l-1}} d x_{i_{l+1}} \cdots d x_{i_{m}} \sum_{r=1}^{n} \xi_{r} d x_{r} \\
& =\sum_{i_{1}=1}^{n} \sum_{i_{2}=1}^{n} \cdots \sum_{i_{m}=1}^{n} d x_{i_{1}} d x_{i_{2}} \cdots d x_{i_{m}}\left\{\alpha_{i_{1} i_{2} \ldots i_{m}}+\sum_{r=1}^{n} \sum_{l=1}^{m} a_{i_{1} \ldots i_{l-1} r i_{l+1} \ldots i_{m}} \xi_{i_{l}}\right\} .
\end{aligned}
$$

Hence

$$
-\alpha_{i_{1} i_{2} \ldots i_{m}}=\sum_{r=1}^{n} \sum_{l=1}^{m} a_{i_{1} i_{2} \ldots i_{l-1} r i_{l+1} \ldots i_{m}} \xi_{i_{l}} .
$$

The equations for the invariants of order zero are obtained by equating to zero the coefficients of the $\xi_{r \mid i_{l}}$ in

$$
X_{0} f \equiv \sum_{i_{1}=1}^{n} \sum_{i_{2}=1}^{i_{1}} \ldots \sum_{i_{m}=1}^{i_{m-1}} \alpha_{i_{1} i_{2} \ldots i_{m}} p_{i_{1} i_{2} \ldots i_{m}} \cdot
$$

\section{§3. Enumeration of the equations.}

There are $n^{2}$ of the quantities $\xi_{r \mid s}$, hence $n^{2}$ equations. The number of variables $p_{i_{1} i_{2} \ldots i_{m}}$ is that of the number of distinct terms in the homogeneous polynomial of degree $m$ in $n$ variables, namely *

$$
(n, m) \equiv \frac{n(n+1) \cdots(n+m-1)}{m !}=\frac{(n+m-1) !}{(n-1) ! m !} .
$$

* Cf. Chrystal, Algebra, vol. 2, p. 12. 
We suppose in what follows that

Now

$$
m>2, \quad n \equiv 2 .
$$

and

$$
(n, m+1)=\frac{n+m}{m+1}(n, m),
$$

But

and

$$
\begin{gathered}
\frac{n+m}{1+m}>1 . \\
\therefore(n, m+1)>(n, m), \\
\therefore(n, m)>(n, 3), \quad m>3, \\
\therefore(n, m)-n^{2}>(n, 3)-n^{2}, \quad m>3 .
\end{gathered}
$$

For

$$
(n, 3)-n^{2}=0, \quad n=2,
$$

and

$$
(n, 3)-n^{2}>0, \quad n>2 \text {. }
$$

$$
\begin{gathered}
(n, 3)-n^{2}=\frac{n(n-1)(n-2)}{6}, \\
\therefore(n, m)-n^{2}=0, \quad n=2, m=3,
\end{gathered}
$$

$$
(n, m)-n^{2}>0, \quad n>2, m \equiv 3 .
$$

Hence, whether the equations of order zero are independent or not, there are certainly, except in the case of the binary cubic, for which $n=2, m=3$, invariants of order zero; and, if the equations of order zero are all independent, there are precisely

invariants of that order.

$$
(n, m)-n^{2}
$$

Consider now the equations of higher order. In passing from the $(\mu-1)$ th extension to the $\mu$ th we add a number of equations equal to the number of derivatives of order $\mu+1$ of the $n$ functions $\xi_{r}$, and a number of variables equal to the number of derivatives of order $\mu$ of the $(n, m)$ functions $a_{i_{1} i_{2} \ldots i_{m}} \cdot$ These numbers are, respectively,

and

$$
n(n, \mu+1)=\frac{n(n+\mu)}{\mu+1}(n, \mu),
$$

$$
(n, m) \cdot(n, \mu) \text {. }
$$


Hence, if the equations of orders $\mu-1$ and $\mu$ are all independent, the number of invariants of order $\mu$ is

$$
\left\{(n, m)-\frac{n(n+\mu)}{\mu+1}\right\}(n, \mu) .
$$

\section{§4. Independence of the equations.}

In the enumeration of the invariants of the quadratic differential forms the independence of the equations was proved step by step in the following manner.

First, it was shown that the equations of order $\mu$ differ from those of order $\mu-1$ in that, first, certain terms are added to those equations, and second, certain equations are annexed to the system. These annexed equations were called the final equations of order $\mu$. It was shown that the variables in these equations appear in the equations of no lower order.

Second, it was shown that if all the equations of order $\mu-1$ are independent, and, if the final equations of order $\mu$ are independent, then all the equations of order $\mu$ are independent.

Third, it was shown that if the final equations of order $\mu-1$ are all independent, then the final equations of order $\mu$ must be so likewise.

Fourth, it was shown that there exists an order $\mu$ for which the final equations are independent, and an order $\mu_{1}$ for which all the equations are independent.

In the present problem the proof follows the same lines. Furthermore, the demonstration of the first three steps in the proof follows almost word for word the demonstration already given for quadratic forms. It will, therefore, be omitted here. In the case of quadratic forms, the two parts of the fourth step required separate demonstration. In the present case the matter is simplified in that we are able to prove the following

Theonem.-The equations of order zero are, for forms of degree $m \equiv 3$ in $n \equiv 2$ variables, all independent.

As the equations of order zero are likewise the final equations of that order, the entire fourth step of the proof is covered by this theorem. In the proof it is convenient, in order to avoid disturbing numerical factors, to write the form as follows :

Then

$$
\phi \equiv \sum_{i_{1}=1}^{n} \sum_{i_{2}=1}^{i_{1}} \cdots \sum_{i_{m}=1}^{i_{m-1}} b_{i_{1} i_{2} \ldots i_{m}} d x_{i_{1}} d x_{i_{2}} \ldots d x_{i_{m}}
$$

$$
X \phi=\sum_{i_{1}=1}^{n} \sum_{i_{2}=1}^{i_{1}} \cdots \sum_{i_{m}=1}^{i_{m-1}}\left(X b_{i_{1} i_{2} \ldots i_{m}}\right) d x_{i_{1}} d x_{i_{2}} d x_{i_{m}}+\sum_{s=1}^{n} \frac{\partial \phi}{\partial\left(d x_{s}\right)} \sum_{r=1}^{n} \xi_{r} d x_{r} .
$$

The coefficient of $\xi_{\mathrm{s}, r}$ is $\left\{\partial \phi / \partial\left(d x_{s}\right)\right\} d x_{r}$. 
Now the equations for the invariants of order zero are obtained by collecting those terms of the second part of the above expression for $X \phi$ which are multiplied by each of the products $d x_{i_{1}} \cdots d x_{i_{m}}$, replacing $d x_{i_{1}} \cdots d x_{i_{m}}$ by

$$
q_{i_{1} \ldots i_{m}} \equiv \frac{\partial f}{\partial\left(b_{i_{1} \ldots i_{m}}\right)},
$$

rearranging the result so as to collect the terms forming the coefficients of the $n^{2}$ quantities $\xi_{s r}$, and then equating these coefficients to zero. Hence these equations may be obtained if in the equations

$$
\frac{\partial \phi}{\partial\left(d x_{s}\right)} d x_{r}=0, \quad(r, s=1,2, \cdots n),
$$

we replace the product $d x_{i_{1}} \cdots d x_{i_{m}}$ by the symbol $q_{i_{1} \ldots i_{m}}$. The matrix of the coefficients, therefore, which is all that is of importance in the independence proof, is the same as the matrix of the coefficients in equations (14).

Consider, next, the discriminant of the form $\phi$. It is the resultant of the $n$ equations

$$
\frac{\partial \phi}{\partial\left(d x_{r}\right)}=0, \quad(r=1,2 \cdots n),
$$

and may be written as a determinant which includes the matrix of $n^{2}$ rows and $(n, m)$ columns arising from the equations

$$
\frac{\partial \phi}{\partial\left(d x_{s}\right)} d x_{r}=0, \quad(r, s=1,2, \cdots n),
$$

together with additional rows and columns.

$B u t$ in the columns annexed to these $n^{2}$ rows the elements are all zero.

Suppose the equations of order zero were not all independent. Then all the determinants of order $n^{2}$ formed from the matrix of (14) vanish. But in the resultant all other minors of order $n^{2}$ formed from these $n^{2}$ rows contain at least one column of zeros and therefore vanish. Hence all minors of order $n^{2}$ formed from these $n^{2}$ rows of the resultant vanish. Therefore the resultant itself vanishes. But this resultant is the discriminant of the form, and by hypothesis it does not vanish. Hence the supposition that the equations of order zero are not all independent leads to a contradiction and the theorem is proved.

It follows, then, that the equations of all orders are independent and that, therefore, the number of invariants of order $\mu$ is precisely the excess of variables added over equations added in passing from order $\mu-1$ to order $\mu$, namely

$$
\left\{(n, m)-\frac{n(n+\mu)}{\mu+1}\right\}(n, \mu) \text {. }
$$


§ 5. Simultaneous forms.

The extension to simultaneous forms is obvious. There are for $p$ forms

$$
(n, \mu)\left\{p(n, m)-\frac{n(n+\mu)}{\mu+1}\right\}=(n, \mu)\left\{p\left[(n, m)-\frac{n(n+\mu)}{\mu+1}\right]\right.
$$

invariants of order $\mu$. Of these

$$
\left.+(p-1) \frac{n(n+\mu)}{\mu+1}\right\}
$$

$$
p\left[(n, m)-\frac{n(n+\mu)}{\mu+1}\right](n, \mu)
$$

are invariants of the $p$ separate forms, while the remaining

$$
(p-1) \frac{n(n+\mu)}{\mu+1}(n, \mu)
$$

are true simultaneous invariants which may be taken as invariants of the $p-1$ pairs of forms obtained by associating any given form with the remaining $p-1$ in succession.

Massachusetts Institute of Technology, Boston, 